\title{
Formulation and Evaluation of Sustained Release Preparation of Ibuprofen Fast-Disintegrating Tablet (FDT)
}

\author{
Kwang Hyeon Kim, Jun-Bom Park, Joo-Hyung Kang, Kun-Hee Lee and Chin-Yang Kang ${ }^{\dagger}$ \\ Department of Pharmacy, Sahm Yook University, Seoul 139-742, Korea \\ (Received December 10, $2010 \cdot$ Revised February 7, $2011 \cdot$ Accepted February 8, 2011)
}

\begin{abstract}
The objective of this study was to enhance the utilitization of Ibuprofen (IBU) by introducing the fast-disintegrating tablet (FDT) form. Presently, IBU is being widely used as a tablet or syrup form. But in contrast to these two formulations, IBU as FDT is not only convenient but also increases the control over the time release of the drug, noted by using Alginate beads. This study was carried out with Sodium Alginate and IBU at the ratios of 1:0, 1:0.5, 1:1, and 1:2 in order to produce a series of beads with different ratios. During the drying process of the beads, talc was added in beads to compare the effects with and without the talc. The final product was scanned with SEM imaging to determine the difference in the surface of the beads. The parameters assessed were the diameter, content assay, dissolution test and effectiveness of time-release. Direct compression method was used to prepare FDT containing IBU bead. The properties of FDT, such as hardness, disintegration time, were investigated. The dissolution profiles of FDT were tested using KP dissolution apparatus 1 (basket method). The results suggest addition of talc and drying the beads made the surface smooth and less vulnerable to clutter into chunks. The size of beads was less than $300 \mu \mathrm{m}$ which did not create a sandy feeling in the mouth. Thus, the beads formulation model made the sustained release of the drug possible, the hardness of FDT $\left(1.25 \sim 1.50 \mathrm{Kg} / \mathrm{cm}^{2}\right)$ was acceptable and all the values of dissolving period were less than 30 seconds. The dissolution profile of FDT was same as that of IBU bead. The efficient dissolution profile and low price of IBU bead containing Sodium Alginate, the FDT formulation prepared from IBU bead can save the expenses and can improve the convenience of application of this drug.
\end{abstract}

Key words - Fast-disintegrating tablet, Sustained release, Alginate bead, Ibuprofen

Orally administered formulations such as tablets, granules, powders and liquid preparations are well accepted as they are convenient for administration, manufactured using simpler technology possess stable physical and chemical properties(Marshall et al., 1990). It is also true that liquid formulations are preferred over solid formulations in patients with swallowing difficulties or those who are less compliant to dosing regimen and dosage time and also requires sufficient quantity of water for swallowing the tablet. The liquid formulations are also associated with many drawbacks like- the need of a measuring tool so as to deliver the exact dose of medication, need for a suitable container for storage as the liquid formulations are sensitive to environmental conditions such as sunlight and humidity. Recently, the fast disintegrating tablets(FDTs) do not require any water to aid swallowing as these formulation are very well disintegrate by the saliva in the oral cavity easily and rather quickly. Thus, formulating the drugs as FDTs could offer many advantages while its manufacturing is also fairly convenient compared to other orally administered

Corresponding Author:

Tel : +82-2-3399-1600, E-mail : kangjy@syu.ac.kr

DOI : 10.4333/KPS.2011.41.1.051 formulations (Simone et al., 2002). FDTs offers added benefits to the patients such as enhanced convenience which leads to better patient compliance, its consumption is independent of the availability of water, it does not require chewing. FDTs are also characterized by an increased drug stability, steady drug release, increased bioavailability and it also offers savings in packaging process compared to liquid formulations (Chang et al., 2000; Dobetti 2000; Bi et al., 1999; Bi et al., 1996; Watanabe et al., 1995; Orjales-Venero et al., 1993).

As these tablets have to be easily disintegrated in the oral cavity, during the manufacture of FDT, it is very critical to maintain the pores in a structured array to facilitate the FDT to absorb the moisture easily. The quantity of disintegrating agent or water-soluble excipients has to be used in optimum to derive at a stable design. Developing an FDT as a sustained release formulation could be an excellent strategy that has all the advantage of FDT and maximize the benefit of the medication intended for treatment.

Among the numerous manufacturing methods for sustainedrelease preparation, one of the methods is using alginic acid beads. Dispersing the sodium alginate into the drug renders it insoluble in water, it unloads in the $\mathrm{CaCl}_{2}$ aqueous solution and the resultant formulation, a sphere is generated (Bodmeir et al., 
1989). Moreover, an alginate is known to sensitively swell in a specific $\mathrm{pH}$. It reswells in the phosphate buffer solution of a $\mathrm{pH} 7.0$ more than the original size, which is contracted back to a bead once dried. The $\mathrm{KCl}-\mathrm{HCl}$ buffer solution of $\mathrm{pH} 1.6$ or the distilled water does not allow re-swelling (Yotsuyanagi et al., 1987). Therefore, when the dried gel bead is administered orally, the physical state remains preserved in the upper GI tract, until it is moved to the small intestine where the swelling occurs and the emission of the contained drug occurs. Using these 2 characters of alginate beads, where the bead size is a micro-unit it is possible to manufacture a sustained release model of FDT.

The formulation of FDT which can continuously release the drug moiety will definitely be a challenge. Microspheres in which the drug is loaded during manufacture are requested to possess sufficient physical strength that it can endure, including the required hardness $\left(1.25 \mathrm{~kg} / \mathrm{cm}^{2} \sim 1.50 \mathrm{~kg} / \mathrm{cm}^{2}\right)$ which is needed for the tablet (Sameer et al., 2009). Since, it has to be rapidly disintegrated on a tongue with each microsphere the size of the disintegrated particle is expected to be small. It should be ensured that the size of the bead particle has to be smaller than $300 \mu \mathrm{m}$ to obviate the sandy feeling in the oral cavity (Jung et al., 2008).

Ibuprofen (IBU) is an anti-inflammatory, antipyretic and analgesic of the NSAID series widely used for its ability to demonstrate rapid drug action with minimal side effects. The existing formulation that are currently available are limited mainly to the syrup, tablet, and capsules (Geisslinger et al., 1994; Geisslinger et al., 1993; Luckow et al., 1992; Fu et al., 1991; Kendall et al., 1990; Gadalla et al., 1989; Small et al., 1989; Greenblatt et al., 1984; Mason 1984), there is a need to upgrade the formulation due to the obvious benefits of FDT.

In this study, we tried to estimate the content after ibuprofen is dispersed into sodium alginic acid aqueous solution it is loaded in the $\mathrm{CaCl}_{2}$ aqueous solution in order to synthesize a bead of size $300 \mu \mathrm{m}$ or less, to confirm if the drug loading has taken place or not, content test of a bead is performed. Sustained release FDT can also be manufactured using the bead so as to enhance the convenience and benefits of taking the medication.

\section{Materials and Methods}

\section{Materials}

Ibuprofen was provided by Hwail Pharma Co., Ltd. (Seoul, South Korea). Sodium alginate was bought from the Junsei Chemical Co., Ltd. (Tokyo, Japan). Calcium chloride, $\beta$ Cyclodextrin, Calcium silicate, Polysorbate 20 from Junsei
Chemical Co., Ltd. (Tokyo, Japan), talc from Hongseong Pharma Co., Ltd. (Seoul, Korea), Lactose and Starch from DaeHeung Pharma Co., Ltd. (Seoul, Korea), Croscarmellose sodium and Magnesium stearate from Hwail Pharma Co., Ltd. (Seoul, South Korea), Ammonia solution, Sodium chloride, Disodium phosphate from SamChun chemical Co., Ltd. (Seoul, Korea) Chloroacetic acid from Acros organics Co. Ltd. (New. Jersey, USA). Acetonitrile, Distilled water obtained from Burdick \& Jackson Co. Ltd. (Ulsan, Korea), Sodium hydroxide from Kanto chemicals Co. (Isehara, Japan), Potassium phosphate, and monobasic Phosphoric acid from Sigma Chemical Co., Ltd. (St. Louis, USA). All the reagents and chemicals used in this study were of extra pure or above grade.

\section{Methods}

Manufacturing Beads and FDT

After manufacturing the $2 \%$ Sodium alginate (SA) aqueous solution, Ibuprofen (IBU) was mixed according to the concentrations (as indicated in Table I) were dispersed into a homogenizer for 30 mines. After unloading the dispersion in $0.1 \mathrm{M} \mathrm{CaCl}_{2}$ aqueous solution through 22 gauge needle and talc was added talc as shown in Table I. It was stirred for 1 hour and dried at $70^{\circ} \mathrm{C}$ for 8 hours (Scheme 1). Dried beads were made after measuring the required quantity as shown in Table II. The preparation was mixed with $\beta$-Cyclodextrin, Lactose, Croscarmellose sodium, Calcium silicate, Starch and Magnesium stearate was added and again mixed and compressed by Rotary Tableting machine (Chamunda Pharma, CPM 03-10) (Ahmedabad, India).

\section{Bead evaluation}

Content test

In each formulation, after measuring the bead corresponding to the IBU $100 \mathrm{mg}$ and finely pulverizing it with a mortar and

Table I. Formulation of beads

\begin{tabular}{cccc}
\hline \hline \multirow{2}{*}{ Formulation } & \multicolumn{3}{c}{ Ingredients } \\
\cline { 2 - 4 } & Alginate & IBU & Talc \\
\hline FN -1 & $2 \mathrm{~g}$ & $0 \mathrm{~g}$ & $\mathrm{X}$ \\
FN -2 & $2 \mathrm{~g}$ & $0 \mathrm{~g}$ & $\mathrm{O}$ \\
FN -3 & $2 \mathrm{~g}$ & $1 \mathrm{~g}$ & $\mathrm{X}$ \\
FN -4 & $2 \mathrm{~g}$ & $1 \mathrm{~g}$ & $\mathrm{O}$ \\
FN -5 & $2 \mathrm{~g}$ & $2 \mathrm{~g}$ & $\mathrm{X}$ \\
FN -6 & $2 \mathrm{~g}$ & $2 \mathrm{~g}$ & $\mathrm{O}$ \\
FN -7 & $2 \mathrm{~g}$ & $4 \mathrm{~g}$ & $\mathrm{X}$ \\
FN -8 & $2 \mathrm{~g}$ & $4 \mathrm{~g}$ & $\mathrm{O}$ \\
\hline
\end{tabular}




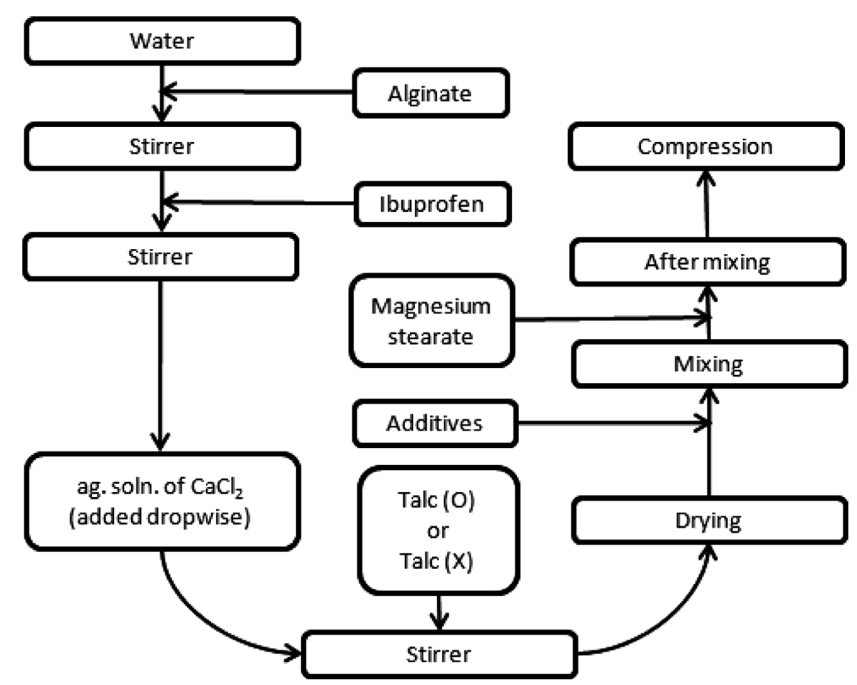

Scheme 1. Schematic diagram of the method to prepare FDT with IBU bead.

pestle, it was passed on to the HPLC mobile phase $50 \mathrm{~mL}$ for 1 hour with sonication following which it was analyzed with HPLC. IBU was detected by a Beckman HPLC system (Fullerton, USA), consisting of a UV detector (168), C18 column (4.6×120 mm, $5 \mu \mathrm{m}$, Waters), pump (126), autosampler (508), column oven (234), and data processor (Karat 32).

Drug loading and entrapment efficiency were also checked as per the equations below:

Drug loading $(\% \mathrm{w} / \mathrm{w})=$

$$
\frac{\text { mass of IBU in bead }}{\text { mass of bead produced }} \times 100
$$

$$
\begin{aligned}
& \text { Entrapment efficiency }(\%)= \\
& \frac{\text { mass of IBU in bead }}{\text { mass of IBU used in formulation }} \times 100
\end{aligned}
$$

\section{SEM Image}

The image of FN-2,7,8 beads were enlarged to $250 \mathrm{x}$ and observed through SEM for the size of a single bead, the texture of the bead surface, the difference of talc existence and nonexistence, dryness etc.

\section{Dissolution test}

By using the bead applicable to Tablet I among the Korean Pharmacopeia dissolution test method according to the first (Rotation sample box method) the phosphate buffer solution (pH 7.2) in $37^{\circ} \mathrm{C}$ as the solvent were released at $50 \pm 2 \mathrm{rpm}$, were performed with the Dissolution tester (Pharma Test, Germany). It was set up in intervals of 5, 10, 20, 30, 60, 90, 120, 180 minutes, a sample of $5 \mathrm{~mL}$ was gathered in an autosampler and analyzed with HPLC.
Table II. Formulation of FDTS

\begin{tabular}{lccc}
\hline \hline & \multicolumn{3}{c}{ FDT Formulation (mg) } \\
\cline { 2 - 4 } & FDT-1 & FDT-2 & FDT-3 \\
\hline IBU & FN-7 & FN-8 & Powder \\
ß-Cyclodextrin & $60 \mathrm{mg}$ & $60 \mathrm{mg}$ & $60 \mathrm{mg}$ \\
Lactose & $20 \mathrm{mg}$ & $20 \mathrm{mg}$ & $20 \mathrm{mg}$ \\
Croscarmellose sodium & $6 \mathrm{mg}$ & $6 \mathrm{mg}$ & $6 \mathrm{mg}$ \\
Calcium silicate & $5 \mathrm{mg}$ & $5 \mathrm{mg}$ & $5 \mathrm{mg}$ \\
Magnesium stearate & $2 \mathrm{mg}$ & $2 \mathrm{mg}$ & $2 \mathrm{mg}$ \\
Starch & $53 \mathrm{mg}$ & $53 \mathrm{mg}$ & $103 \mathrm{mg}$ \\
\hline
\end{tabular}

(tablet weight $=300 \mathrm{mg}$ )

Table III. Constituent of simulated salivia fluid

\begin{tabular}{cc}
\hline \hline & Constituent (per liter of distilled water) \\
\hline $\mathrm{Na}_{2} \mathrm{HPO}_{4}$ & $2.38 \mathrm{~g}$ \\
$\mathrm{KH}_{2} \mathrm{PO}_{4}$ & $0.19 \mathrm{~g}$ \\
$\mathrm{Na}_{2} \mathrm{HPO}_{4}$ & $8.00 \mathrm{~g}$ \\
\hline
\end{tabular}

(pH adjusted to 6.76 with phosphoric acid)

\section{FDT evaluation}

Weight, Hardness

Each FDT manufactured tablets were measured according to each Formulation with 3T, the weight and hardness.

\section{Disintegration test}

A tester was hung on the contraction and placed in a beaker. For the smooth movement of the dip up and down 29 32 times round trip and amplitude $53 \sim 57 \mathrm{~mm}$ for 1 minute was controlled according to KP IX. Artificial saliva was used as the integration solution (Table III).

\section{Dissolution test}

We tested on the same condition as the dissolution test of "Bead evaluation".

\section{Results and Discussion}

\section{IBU Bead}

\section{Drug loading}

The Drug loading value is displayed in Table IV. FN-1, FN2 are beads manufactured with the sodium alginate (SA). Therefore, drug loading value is $0 \%$. The drug loading value is enhanced by the (FN-3, FN-4), (FN-5, FN-6) and (FN-7, FN8 ). In addition to this, the IBU/SA value used in formulation is shown to increase. 
Table IV. Drug loading of IBU bead

\begin{tabular}{cc}
\hline \hline Formulation & Drug loading (\%) \\
\hline FN-1 & $0.00 \%$ \\
FN-2 & $0.00 \%$ \\
FN-3 & $16.43 \%$ \\
FN-4 & $17.57 \%$ \\
FN-5 & $28.24 \%$ \\
FN-6 & $29.46 \%$ \\
FN-7 & $61.23 \%$ \\
FN-8 & $62.85 \%$ \\
\hline
\end{tabular}

Table V. Entrapment efficiency of IBU bead

\begin{tabular}{cc}
\hline \hline Formulation & Entrapment efficiency (\%) \\
\hline FN-3 & $49.61 \%$ \\
FN-4 & $52.71 \%$ \\
FN-5 & $56.48 \%$ \\
FN-6 & $58.92 \%$ \\
FN-7 & $91.84 \%$ \\
FN-8 & $94.28 \%$ \\
\hline
\end{tabular}

\section{Entrapment efficiency}

The Entrapment efficiency value is shown in Table V. As to FN-1, and FN-2, IBU does not enter the bead and there is no entrapment efficiency value. The entrapment efficiency value is remarkably higher than FN-3, FN-4, FN-5, and FN-6 from FN-7, and FN-8 to about 1.73 times. These 2 formulations will be used in the FDT manufacture.

\section{SEM Image}

The SEM Images of FN-2, FN-7, and FN-8 were compared in order to confirm the form of the bead in the IBU loading, the affect of difference in talc addition, existence and nonexistence reaches to the bead type in the bead drying procedure, and the size and surface feature of a bead. In Figure 1(a), in this bead since IBU is not loaded, the surface appears smooth. In the bead which is loaded with IBU, (b) and (c) we can visualize the IBU crystals on the surface. Talc was added and as for the dry FN-2, and FN-8 the surface remained smooth and it was equally spherical in shape, on the other hand, the shape of the bead without talc showed a surface like the dry FN-7, that was rough and the shape of a beads were not fixed. And it was noted that by adding talc the beads remained dry, and beads appeared together. As FDT has to be manufactured with a bead, it has to be $300 \mu \mathrm{m}$ or less in to ensure that there is no sandy feeling in mouth. Figure 1 displays the image where the size can be confirmed that it is $300 \mu \mathrm{m}$ or less.
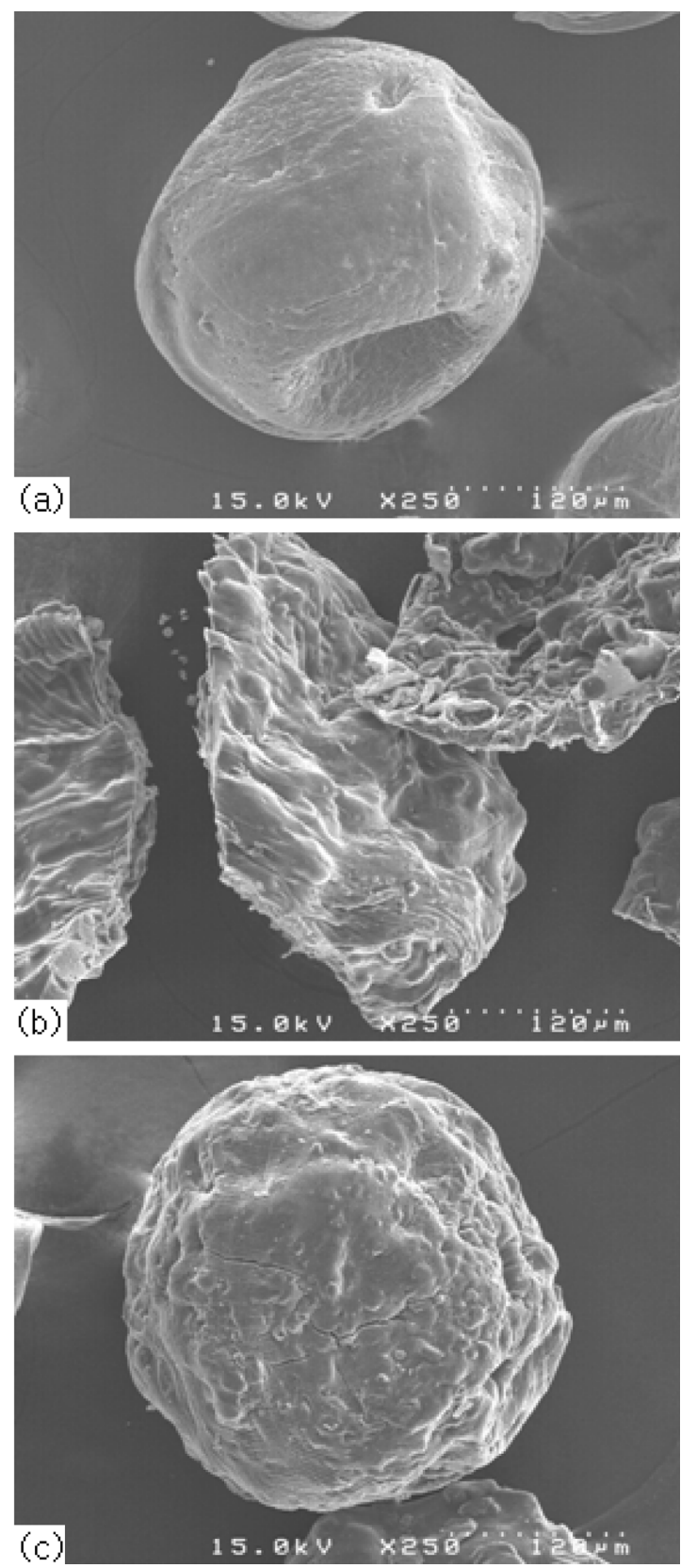

Figure 1. Representative scanning electron micrographs of bead formulation by: (a) FN-2, (b) FN-7 and (c) FN-8.

\section{Dissolution test}

The IBU powder, FN-7, and FN-8 were made with the comparison dissolution. In order to confirm the IBU emission change by existence of talc which is added when dry and the IBU emission change by SA. In the IBU powder, $80 \%$ was released in 30 minutes. But $80 \%$ of IBU was released in 120 minutes from FN-7 and FN-8. Thus it could be inferred that talc can control the initial burst of IBU through the SA bead. The dissolution type of FN-7 and FN-8 showed no difference 


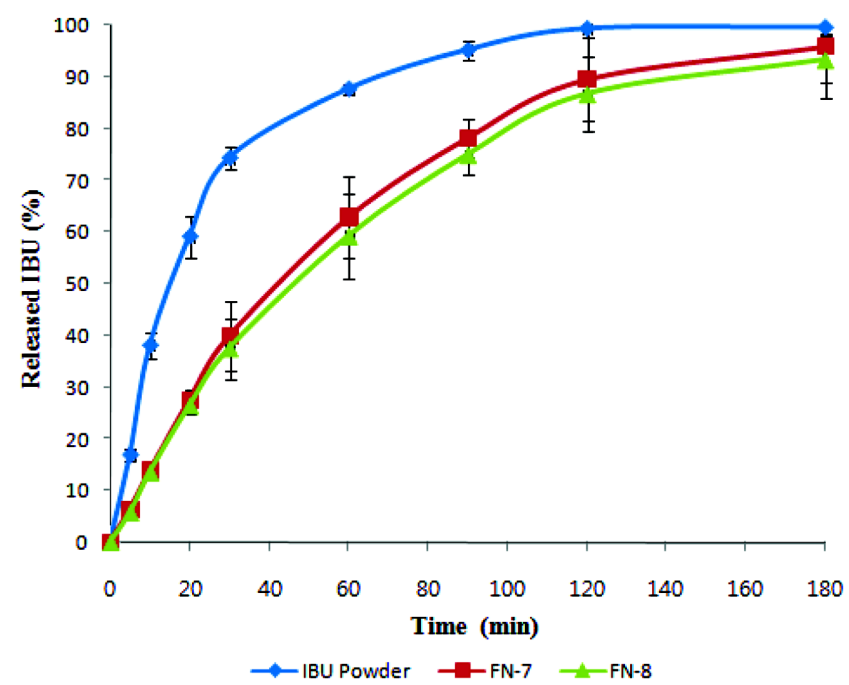

Figure 2. Dissolution profile of IBU powder, FN-7 and FN-8.

and it was dry, effect of talc addition and the release of IBU showed no correlation (Figure 2).

\section{IBU FDT}

\section{Weight}

FDT was compressed with the formulation that is shown in Table II. And it was randomly selected with $3 \mathrm{~T}$ and the weight was measured. The measurement result average weight was $300 \pm 2 \mathrm{mg}$. (Figure 3).

\section{Hardness}

FDT was compressed with the formulation that it shows with the Table II and it was randomly selected with $3 \mathrm{~T}$ and the hardness was measured. The measurement result average hardness was $1.26 \sim 1.45 \mathrm{Kg} / \mathrm{cm}^{2}$. This is the value in which it enters into $1.25 \sim 1.50 \mathrm{Kg} / \mathrm{cm}^{2}$, that fits the hardness of FDT (Figure 4).

\section{Disintegration test}

Figure 5 shows the results of the disintegration test in the artificial saliva solution, considering FDT of 3Tformulation.

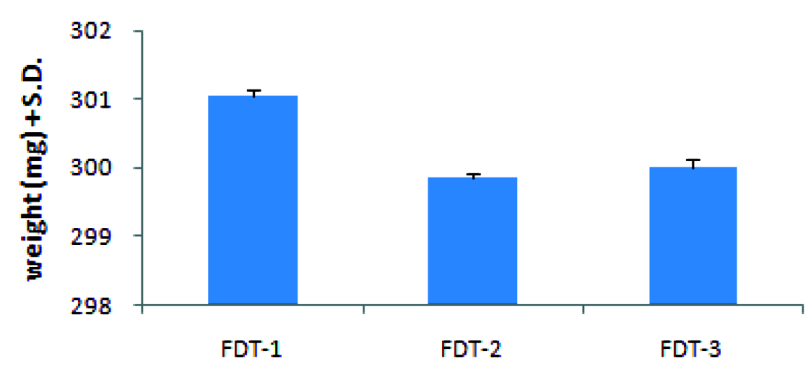

Figure 3. Weight profile of FDT-1, FDT-2 and FDT-3.

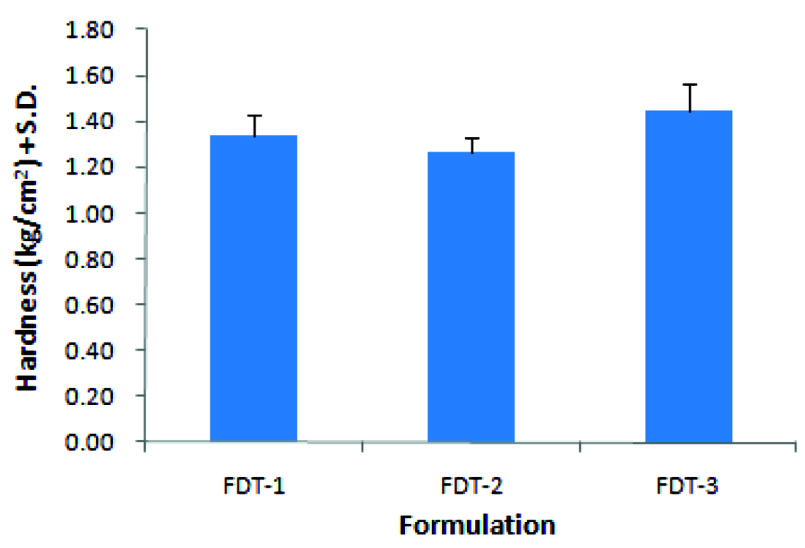

Figure 4. Hardness profile of FDT-1, FDT-2 and FDT-3.

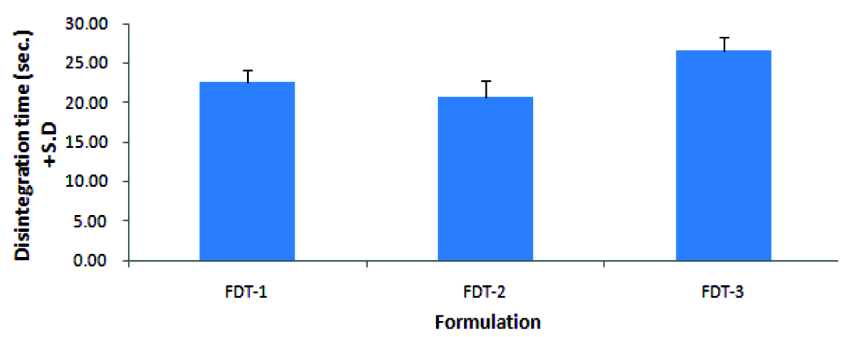

Figure 5. Disintegration time profile of FDT-1, FDT-2 and FDT-3.

The time taken for disintegration was in acceptable range with a buffer less than 30 seconds. The disintegration time of FDT3 was longer than FDT- 1 and FDT- 2 by $4 \sim 6$ seconds. This phenomenon could be attributed to higher starch content $(50 \mathrm{mg})$ in FDT-3. The disintegration time of FDT-3 was observed to be 3 minutes longer than FDT-1 and FDT-2.

\section{Dissolution test}

In the dissolution test, it was confirmed that SA was suc-

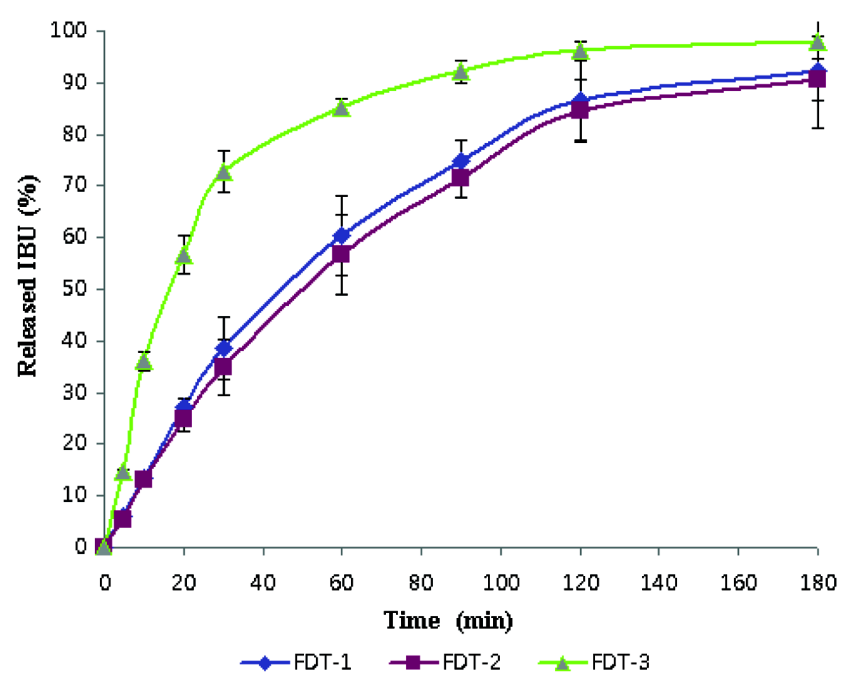

Figure 6. Dissolution profile of FDT-1, FDT-2 and FDT-3. 
cessful in bringing a delay in IBU release. The results of FDT1, FDT-2, and FDT-3 were comparable in dissolution indicating that IBU release delay effect was seen constantly even after tabletizing a bead with FDT. The dissolution type of FDT1 and FDT- 2 were similar in both the beads. When compared with FDT-3, it was confirmed that the initial release of IBU in FDT-1 and FDT-2 was suppressed (Figure 6). Although FDT was manufactured with a bead, it could be demonstrated that the IBU dissolution type at the bead was similar to that maintained in FDT. It is observed that the volume of IBU that is dissolved from all sampling points is generally reduced than the bead. This could be due to the fact that the applied tabletting pressure could have an influence over the bead.

\section{Conclusions}

In this study, the bead was made using SA and confirmed that a controlled-release of IBU, was possible. The most suitable SA: IBU rate was pre-determined for the manufacture of the bead. And in the bead drying procedure, the difference of talc addition existence and nonexistence was confirmed. The Fast-disintegrating tablet which provides the convenience of taking the medicine and enhanced profitability due to simpler manufacturing process (the low cost of SA, and tabletting through direct compression) as the produced bead was compressed. The release type in a bead and the release type in FDT were compared so that we could confirm whether the controlled-release of IBU was still maintained after the FDT tabletting. $3 \mathrm{~T}$ or the amounts corresponding to $3 \mathrm{~T}$ were taken in the dissolution test in each formulation. As soon as the dissolution test was completed and in order to minimize any change in the stability of a drug, we analyzed it in HPLC. The experimental results were derived and conclusions can be drawn.

1. This study confirmed that IBU dissolves well in 200ppm concentration in the $1 \%$ Tween 20 dissolution solution. IBU dissolved well in a dissolution solution at a minimum 100ppm concentration in order to progress the dissolution test. Therefore, we could use the $1 \%$ Tween 20 phosphate buffer solution as a dissolution solution.

2. The Drug loading value and entrapment efficiency value were found after the bead manufacture. Of the 2 parameters, it was observed that in FN-7, and FN-8 showed higher values than any other formulation and thus was considered as the most suitable SA: IBU rate 1: 2 in the bead manufacture.

3. The bead containing talc was observed through the SEM in the bead drying was smoother and the shape and size confirmed that it is equal and spherical in shape. The existence or nonexistence of talc did not influence the dissolution type in the dissolution test result bead drying. Since, it can get the bead in a fixed size and shape, the duration for the drying procedure was lessened, and it is desirable that talc is added on the bead only during drying procedure.

4. The study also confirmed that SA suppresses the initial release of IBU, as observed in the dissolution test of the bead and dissolution test of FDT. It was confirmed that there was no difference between the IBU gush type of in bead and FDT. Therefore, the feasibility for manufacturing the sustainedrelease FDT with a bead is a possible option. It is possible to apply this technique to other poorly water soluble drugs as well.

\section{References}

Bi, Y., Sunada, H., Yonezawa, Y., Danjo, K., 1999. Evaluation of rapidly disintegrating tablets prepared by a direct compression method. Drug Dev. Ind. Pharm. 25, 571-581.

Bi Y., Sunada H., Yonezawa Y., Danjo K., Otsuka A., Iida K., 1996. Preparation and evaluation of a compressed tablet rapidly disintegrating in the oral cavity. Chem. Pharm. Bull. 44, 2121-2127.

Bodmeir R., Paeratakul O., 1989. Spherical agglomerate of waterinsoluble drugs, J. Pharm. Sci., 78, 964-966.

Chang R-K., Guo X., Burnside B.A., Couch R.A., 2000. Fast-dissolving tablets. Pharm. Technol. Eur., 12, 52-58.

Dobetti L., 2000. Fast-melting tablets: developments and technolgies. Pharm. Technol. Eur., 12, 32-42.

Fu C.H., Melethil S., Mason W.D., 1991. Pharmacokinetics of aspirin in rats and the effect of buffer, J. Pharmacokinet. Biopharm., 19, 157-173.

Gadalla M.A., Ismail A.A., El-Hameed M.H.Abd., 1989. In vivo evaluation of different types of commercial aspirin tablets, Drug Dev. Ind. Pharm., 15, 447-472.

Geisslinger G., Manzel S., Zoller O., Cheng Z.H., Brune K., 1994. Absorption and distribution of ibuprofen and acetylsalicylic acid formulations, Drug Invest., 1, 52-55.

Geisslinger G., Menel S., Wissel K., Brune K., 1993. Single dose pharmacokinetics of different formulations of ibuprofen and aspirin, Drug Invest. 5, 238-242.

Greenblatt D.J., Abernethy D.R., Matlis R., Harmatz J.S., 1984. Absorption and distribution of ibuprofen in the elderly arthritis rheumatitis, Clin. Pharm., 27, 1066-1069.

Jung S. H., Park K., 2008. Development of sustained release fastdisintegrating tablets using various polymer-coated ionexchange resin complexes, Int. J. Pharm. 353, 195-204.

Kendall M.J., Jubb R., Bird H.A., Gallez P.Le., Rau R., 1990. Pharmacokinetic comparison of ibuprofen sustained-release tablets given to young and elderly patients. J. Clin. Pharm. Ther., 15, 35-40.

Luckow V., Krammer R., Traub R., 1992. Investigation of com- 
parative bioavailability of two different ibuprofen grannules, Arzneim-Forsch., 42, 1339-1342.

Marshall K., Rudnic E.M., 1990. Tablet dosage forms. In: Modern Pharmaceutics. Marcel Dekker Inc., New York.

Mason W.D., 1984. Kinetics of aspirin absorption following oral administration of six aqueous solutions with different buffer capacities, J. Pharm. Sci., 73, 1258-1261.

Orjales-Venero A., Beascoa-Alzola E., Lucero de Pablo M.L., 1993. Process to prepare water-dispersable tablets containing diclofenac. EP., 0, 599.

Sameer G.L., Yi-Ying Y., Ajay K.B., 2009. Effects of disinte- gration-promoting agent, lubricants and moisture treatment on optimized fast disintegrating tablets, Int. J. Pharm. 365, 4-11. Simone S., Peter C.S., 2002. Fast dispersible ibuprofen tablets, EUR. J. Pharm. Sci., 15, 295-305.

Small R.E., Wood J.H., 1989. Influence of racial differences on effects of ranitidine and cimetidine in ibuprofen pharmacokinetics, Clin. Pharm., 8, 471-472.

Watanabe Y., Koizumi K., Zama Y., Kiriyama M., Matsumoto Y., Matsumoto M., 1995. New compressed tablet rapidly disintegrating in saliva in the mouth using crystalline cellulose and a disintegrant. Biol. Pharm. Bull. 18, 1308-1310. 